\title{
Article
}

\section{Existence results for a class of nonlinear degenerate $(p, q)$-biharmonic operators}

\section{Albo Carlos Cavalheiro}

Department of Mathematics, State University of Londrina, Londrina-86057-970, PR-Brazil.; accava@gmail.com Received: 3 September 2020; Accepted: 3 October 2020; Published: 8 October 2020.

Abstract: In this paper we are interested in the existence of solutions for Navier problem associated with the degenerate nonlinear elliptic equations in the setting of the weighted Sobolev spaces.

Keywords: Degenerate nonlinear elliptic equations, weighted Sobolev spaces.

MSC: 35J70, 35J60, 35J30.

\section{Introduction}

I

$\mathrm{n}$ this paper, we prove the existence of (weak) solutions in the weighted Sobolev space $X=$ $W^{2, p}(\Omega, \omega) \cap W_{0}^{1, p}(\Omega, \omega)$ (see Definition 3 and Definition 4 ) for the Navier problem

$$
\begin{cases}L u(x)=f_{0}(x)-\sum_{j=1}^{n} D_{j} f_{j}(x), & \text { in } \Omega, \\ u(x)=\Delta u(x)=0, & \text { on } \partial \Omega,\end{cases}
$$

where $L$ is the partial differential operator

$$
L u(x)=\Delta\left[\omega(x)\left(|\Delta u|^{p-2} \Delta u+|\Delta u|^{q-2} \Delta u\right)\right]-\sum_{j=1}^{n} D_{j}\left[\omega(x) \mathcal{A}_{j}(x, u(x), \nabla u(x))\right],
$$

where $D_{j}=\partial / \partial x_{j}, \Omega$ is a bounded open set in $\mathbb{R}^{n}, \omega$ is a weight function, $\Delta$ is the usual Laplacian operator, $2 \leq q<p<\infty$ and the functions $\mathcal{A}_{j}: \Omega \times \mathbb{R} \times \mathbb{R}^{n} \rightarrow \mathbb{R}(j=1, \ldots, n)$ satisfying the following conditions:

(H1) $x \mapsto \mathcal{A}_{j}(x, \eta, \xi)$ is measurable on $\Omega$ for all $(\eta, \xi) \in \mathbb{R} \times \mathbb{R}^{n},(\eta, \xi) \mapsto \mathcal{A}_{j}(x, \eta, \xi)$ is continuous on $\mathbb{R} \times \mathbb{R}^{n}$ for almost all $x \in \Omega$.

(H2) there exist a constant $\theta_{1}>0$ such that

$$
\left[\mathcal{A}(x, \eta, \xi)-\mathcal{A}\left(x, \eta^{\prime}, \xi^{\prime}\right)\right] \cdot\left(\xi-\xi^{\prime}\right) \geq \theta_{1}\left|\xi-\xi^{\prime}\right|^{p},
$$

whenever $\xi, \xi^{\prime} \in \mathbb{R}^{n}, \xi \neq \xi^{\prime}$, where $\mathcal{A}(x, \eta, \xi)=\left(\mathcal{A}_{1}(x, \eta, \xi), \ldots, \mathcal{A}_{n}(x, \eta, \xi)\right)$ (where a dot denote here the Euclidian scalar product in $\mathbb{R}^{n}$ ).

(H3) $\mathcal{A}(x, \eta, \xi) . \xi \geq \lambda_{1}|\xi|^{p}$, where $\lambda_{1}$ is a positive constant.

(H4) $|\mathcal{A}(x, \eta, \xi)| \leq K_{1}(x)+h_{1}(x)|\eta|^{p / p^{\prime}}+h_{2}(x)|\xi|^{p / p^{\prime}}$, where $K_{1}, h_{1}$ and $h_{2}$ are positive functions, with $h_{1}$, $h_{2} \in L^{\infty}(\Omega)$, and $K_{1} \in L^{p^{\prime}}(\Omega, \omega)$ (with $1 / p+1 / p^{\prime}=1$ ).

By a weight, we shall mean a locally integrable function $\omega$ on $\mathbb{R}^{n}$ such that $0<\omega(x)<\infty$ for a.e. $x \in \mathbb{R}^{n}$. Every weight $\omega$ gives rise to a measure on the measurable subsets on $\mathbb{R}^{n}$ through integration. This measure will be denoted by $\mu$. Thus, $\mu(E)=\int_{E} \omega(x) d x$ for measurable sets $E \subset \mathbb{R}^{n}$.

In general, the Sobolev spaces $W^{k, p}(\Omega)$ without weights occur as spaces of solutions for elliptic and parabolic partial differential equations. In the particular case where $p=q=2$ and $\omega \equiv 1$, we have the equation

$$
\Delta^{2} u-\sum_{j=1}^{n} D_{j} \mathcal{A}_{j}(x, u, \nabla u)=f,
$$


where $\Delta^{2} u$ is the biharmonic operator. If $p=q, \omega \equiv 1$ and $\mathcal{A}(x, \eta, \xi)=|\xi|^{p-2} \xi$, we have the equation

$$
\Delta\left(|\Delta|^{p-2} \Delta u\right)-\operatorname{div}\left(|\nabla u|^{p-2} \nabla u\right)=f .
$$

Biharmonic equations appear in the study of mathematical model in several real-life processes as, among others, radar imaging (see [1]) or incompressible flows (see [2]).

For degenerate partial differential equations, i.e., equations with various types of singularities in the coefficients, it is natural to look for solutions in weighted Sobolev spaces (see [3-6]). In various applications, we can meet boundary value problems for elliptic equations whose ellipticity is disturbed in the sense that some degeneration or singularity appears. There are several very concrete problems from practice which lead to such differential equations, e.g. from glaceology, non-Newtonian fluid mechanics, flows through porous media, differential geometry, celestial mechanics, climatology, petroleum extraction and reaction-diffusion problems (see some examples of applications of degenerate elliptic equations in $[7,8]$ ).

A class of weights, which is particularly well understood, is the class of $A_{p}$-weights (or Muckenhoupt class) that was introduced by B. Muckenhoupt (see [9]). These classes have found many useful applications in harmonic analysis (see [10]). Another reason for studying $A_{p}$-weights is the fact that powers of distance to submanifolds of $\mathbb{R}^{n}$ often belong to $A_{p}$ (see [11]). There are, in fact, many interesting examples of weights (see [12] for p-admissible weights).

In the non-degenerate case (i.e. with $\omega(x) \equiv 1$ ), for all $f \in L^{p}(\Omega)$, the Poisson equation associated with the Dirichlet problem

$$
\begin{cases}-\Delta u=f(x), & \text { in } \Omega \\ u(x)=0, & \text { on } \partial \Omega\end{cases}
$$

is uniquely solvable in $W^{2, p}(\Omega) \cap W_{0}^{1, p}(\Omega)$ (see [13]), and the nonlinear Dirichlet problem

$$
\begin{cases}-\Delta_{p} u=f(x), & \text { in } \Omega \\ u(x)=0, & \text { on } \partial \Omega\end{cases}
$$

is uniquely solvable in $W_{0}^{1, p}(\Omega)$ (see [14]), where $\Delta_{p} u=\div\left(|\nabla u|^{p-2} \nabla u\right)$ is the p-Laplacian operator. In the degenerate case, the weighted p-Biharmonic operator has been studied by many authors (see [15] and the references therein), and the degenerated p-Laplacian was studied in [6].

The following theorem will be proved in Section 3.

Theorem 1. Let $2 \leq q<p<\infty$ and assume (H1)-(H4). If $\omega \in A_{p}, \frac{f_{j}}{\omega} \in L^{p^{\prime}}(\Omega, \omega)(j=0,1, \ldots, n)$ then the problem (P) has a unique solution $u \in X=W^{2, p}(\Omega, \omega) \cap W_{0}^{1, p}(\Omega, \omega)$. Moreover, we have

$$
\|u\|_{X} \leq \frac{1}{\gamma^{p^{\prime} / p}}\left(C_{\Omega}\left\|f_{0} / \omega\right\|_{L^{p^{\prime}}(\Omega, \omega)}+\sum_{j=1}^{n}\left\|f_{j} / \omega\right\|_{L^{p^{\prime}}(\Omega, \omega)}\right)^{p^{\prime} / p}
$$

where $\gamma=\min \left\{\lambda_{1}, 1\right\}$ and $C_{\Omega}$ is the constant in Theorem 3.

\section{Definitions and basic results}

Let $\omega$ be a locally integrable nonnegative function in $\mathbb{R}^{n}$ and assume that $0<\omega<\infty$ almost everywhere. We say that $\omega$ belongs to the Muckenhoupt class $A_{p}, 1<p<\infty$, or that $\omega$ is an $A_{p}$-weight, if there is a constant $C=C_{p, \omega}$ such that

$$
\left(\frac{1}{|B|} \int_{B} \omega(x) d x\right)\left(\frac{1}{|B|} \int_{B} \omega^{1 /(1-p)}(x) d x\right)^{p-1} \leq C,
$$

for all balls $B \subset \mathbb{R}^{n}$, where $|$.$| denotes the n$-dimensional Lebesgue measure in $\mathbb{R}^{n}$. If $1<q \leq p$, then $A_{q} \subset A_{p}$ (see $[10,12,16]$ for more information about $A_{p}$-weights). The weight $\omega$ satisfies the doubling condition if there exists a positive constant $C$ such that $\mu(B(x ; 2 r)) \leq C \mu(B(x ; r))$, for every ball $B=B(x ; r) \subset \mathbb{R}^{n}$, where $\mu(B)=$ $\int_{B} \omega(x) d x$. If $\omega \in A_{p}$, then $\mu$ is doubling (see Corollary 15.7 in [12]).

As an example of $A_{p}$-weight, the function $\omega(x)=|x|^{\alpha}, x \in \mathbb{R}^{n}$, is in $A_{p}$ if and only if $-n<\alpha<n(p-1)$ (see Corollary 4.4, Chapter IX in [10]). 
If $\omega \in A_{p}$, then

$$
\left(\frac{|E|}{|B|}\right)^{p} \leq C \frac{\mu(E)}{\mu(B)}
$$

whenever $B$ is a ball in $\mathbb{R}^{n}$ and $E$ is a measurable subset of $B$ (see 15.5 strong doubling property in [12]). Therefore, if $\mu(E)=0$ then $|E|=0$. The measure $\mu$ and the Lebesgue measure $|$.$| are mutually absolutely continuous, i.e.,$ they have the same zero sets $(\mu(E)=0$ if and only if $|E|=0)$; so there is no need to specify the measure when using the ubiquitous expression almost everywhere and almost every, both abbreviated a.e..

Definition 1. Let $\omega$ be a weight, and let $\Omega \subset \mathbb{R}^{n}$ be open. For $0<p<\infty$ we define $L^{p}(\Omega, \omega)$ as the set of measurable functions $f$ on $\Omega$ such that

$$
\|f\|_{L^{p}(\Omega, \omega)}=\left(\int_{\Omega}|f(x)|^{p} \omega(x) d x\right)^{1 / p}<\infty .
$$

If $\omega \in A_{p}, 1<p<\infty$, then $\omega^{-1 /(p-1)}$ is locally integrable and we have $L^{p}(\Omega, \omega) \subset L_{\text {loc }}^{1}(\Omega)$ for every open set $\Omega$ (see Remark 1.2.4 in [17]). It thus makes sense to talk about weak derivatives of functions in $L^{p}(\Omega, \omega)$.

Definition 2. Let $\Omega \subset \mathbb{R}^{n}$ be a bounded open set, $1<p<\infty, k$ be a nonnegative integer and $\omega \in A_{p}$. We shall denote by $W^{k, p}(\Omega, \omega)$, the weighted Sobolev spaces, the set of all functions $u \in L^{p}(\Omega, \omega)$ with weak derivatives $D^{\alpha} u \in L^{p}(\Omega, \omega), 1 \leq|\alpha| \leq k$. The norm in the space $W^{k, p}(\Omega, \omega)$ is defined by

$$
\|u\|_{W^{k, p}(\Omega, \omega)}=\left(\int_{\Omega}|u|^{p} \omega d x+\sum_{1 \leq|\alpha| \leq k} \int_{\Omega}\left|D^{\alpha} u\right|^{p} \omega d x\right)^{1 / p} .
$$

If $\omega \in A_{p}$, then $W^{k, p}(\Omega, \omega)$ is the closure of $C^{\infty}(\Omega)$ with respect to the norm (1) (see Corollary 2.1.6 in [17]). We also define the space $W_{0}^{k, p}(\Omega, \omega)$ as the closure of $C_{0}^{\infty}(\Omega)$ with respect to the norm (1). We have that the spaces $W^{k, p}(\Omega, \omega)$ and $W_{0}^{k, p}(\Omega, \omega)$ are Banach spaces.

The space $W_{0}^{1, p}(\Omega, \omega)$ is the closure of $C_{0}^{\infty}(\Omega)$ with respect to the norm (1). Equipped with this norm, $W_{0}^{1, p}(\Omega, \omega)$ is a reflexive Banach space (see [18] for more information about the spaces $W^{1, p}(\Omega, \omega)$ ). The dual of space $W_{0}^{1, p}(\Omega, \omega)$ is the space

$$
\left[W_{0}^{1, p}(\Omega, \omega)\right]^{*}=\left\{T=f_{0}-\operatorname{div}(F), F=\left(f_{1}, \ldots, f_{n}\right): \frac{f_{j}}{\omega} \in L^{p^{\prime}}(\Omega, \omega), j=0,1, \ldots, n\right\} .
$$

It is evident that a weight function $\omega$ which satisfies $0<c_{1} \leq \omega(x) \leq c_{2}$ for $x \in \Omega$ (where $c_{1}$ and $c_{2}$ are constants), give nothing new (the space $W_{0}^{1, p}(\Omega, \omega)$ is then identical with the classical Sobolev space $W_{0}^{1, p}(\Omega)$ ). Consequently, we shall be interested above all in such weight functions $\omega$ which either vanish somewhere in $\bar{\Omega}$ or increase to infinity (or both).

In this paper we use the following results.

Theorem 2. Let $\omega \in A_{p}, 1<p<\infty$, and let $\Omega$ be a bounded open set in $\mathbb{R}^{n}$. If $u_{m} \rightarrow u$ in $L^{p}(\Omega, \omega)$ then there exist a subsequence $\left\{u_{m_{k}}\right\}$ and a function $\Phi \in L^{p}(\Omega, \omega)$ such that

(i) $u_{m_{k}}(x) \rightarrow u(x), m_{k} \rightarrow \infty$ a.e. on $\Omega$;

(ii) $\left|u_{m_{k}}(x)\right| \leq \Phi(x)$ a.e. on $\Omega$.

Proof. The proof of this theorem follows the lines of Theorem 2.8.1 in [19].

Theorem 3. (The weighted Sobolev inequality) Let $\Omega$ be an open bounded set in $\mathbb{R}^{n}$ and $\omega \in A_{p}(1<p<\infty)$. There exist constants $C_{\Omega}$ and $\delta$ positive such that for all $u \in W_{0}^{1, p}(\Omega, \omega)$ and all $k$ satisfying $1 \leq k \leq n /(n-1)+\delta$,

$$
\|u\|_{L^{k p}(\Omega, \omega)} \leq C_{\Omega}\||\nabla u|\|_{L^{p}(\Omega, \omega)} .
$$


Proof. Its suffices to prove the inequality for functions $u \in C_{0}^{\infty}(\Omega)$ (see Theorem 1.3 in [20]). To extend the estimates (2) to arbitrary $u \in W_{0}^{1, p}(\Omega, \omega)$, we let $\left\{u_{m}\right\}$ be a sequence of $C_{0}^{\infty}(\Omega)$ functions tending to $u$ in $W_{0}^{1, p}(\Omega, \omega)$. Applying the estimates (2) to differences $u_{m_{1}}-u_{m_{2}}$, we see that $\left\{u_{m}\right\}$ will be a Cauchy sequence in $L^{k p}(\Omega, \omega)$. Consequently the limit function $u$ will lie in the desired spaces and satisfy (2).

Lemma 1. Let $1<p<\infty$.

(a) There exists a constant $\alpha_{p}>0$ such that

$$
\left.|| x\right|^{p-2} x-|y|^{p-2} y\left|\leq \alpha_{p}\right| x-y \mid(|x|+|y|)^{p-2},
$$

for all $x, y \in \mathbb{R}^{n}$;

(b) There exist two positive constants $\beta_{p}, \gamma_{p}$ such that for every $x, y \in \mathbb{R}^{n}$

$$
\beta_{p}(|x|+|y|)^{p-2}|x-y|^{2} \leq\left(|x|^{p-2} x-|y|^{p-2} y\right) \cdot(x-y) \leq \gamma_{p}(|x|+|y|)^{p-2}|x-y|^{2} .
$$

Proof. See [14], Proposition 17.2 and Proposition 17.3.

Definition 3. We denote by $X=W^{2, p}(\Omega, \omega) \cap W_{0}^{1, p}(\Omega, \omega)$ with the norm

$$
\|u\|_{X}=\left(\int_{\Omega}|\nabla u|^{p} \omega d x+\int_{\Omega}|\Delta u|^{p} \omega d x\right)^{1 / p} .
$$

Definition 4. We say that an element $u \in X=W^{2, p}(\Omega, \omega) \cap W_{0}^{1, p}(\Omega,, \omega)$ is a (weak) solution of problem (P) if

$$
\begin{aligned}
& \int_{\Omega}|\Delta u|^{p-2} \Delta u \Delta \varphi \omega d x+\int_{\Omega}|\Delta u|^{q-2} \Delta u \Delta \varphi \omega d x+\sum_{j=1}^{n} \int_{\Omega} \mathcal{A}_{j}(x, u(x), \nabla u(x)) D_{j} \varphi(x) \omega(x) d x \\
& =\int_{\Omega} f_{0}(x) \varphi(x) d x+\sum_{j=1}^{n} \int_{\Omega} f_{j}(x) D_{j} \varphi(x) d x
\end{aligned}
$$

for all $\varphi \in X$.

Remark 1. If $0<\eta<p<\infty$ then, by Hölder's inequality,

$$
\|u\|_{L^{\eta}(\Omega, \omega)} \leq C_{p, \eta}\|u\|_{L^{p}(\Omega, \omega)}
$$

where $C_{p, \eta}=\left(\int_{\Omega} \omega d x\right)^{(p-\eta) / p \eta}=\|\omega\|_{L^{p /(p-\eta)}(\Omega)}^{1 / \eta}$. In fact,

$$
\begin{aligned}
\|u\|_{L^{\eta}(\Omega, \omega)}^{\eta} & =\int_{\Omega}|u|^{\eta} \omega d x \\
& \leq\left(\int_{\Omega}|u|^{\eta p / \eta} \omega d x\right)^{\eta / p}\left(\int_{\Omega} \omega^{p /(p-\eta)} d x\right)^{(p-\eta) / p} \\
& =\|u\|_{L^{p}(\Omega, \omega)}^{\eta}\|\omega\|_{L^{p /(p-\eta)}(\Omega)} .
\end{aligned}
$$

\section{Proof of Theorem 1}

The basic idea is to reduce the Problem (P) to an operator equation $A u=T$ and apply the theorem below.

Theorem 4. Let $A: X \rightarrow X^{*}$ be a monotone, coercive and hemicontinuous operator on the real, separable, reflexive Banach space $X$. Then the following assertions hold:

(a) For each $T \in X^{*}$ the equation $A u=T$ has a solution $u \in X$;

(b) If the operator $A$ is strictly monotone, then equation $A u=T$ is uniquely solvable in $X$.

Proof. See Theorem 26.A in [21]. 
To prove Theorem 1 , we define $B, B_{1}, B_{2}, B_{3}: X \times X \rightarrow \mathbb{R}$ and $T: X \rightarrow \mathbb{R}$ by

$$
\begin{aligned}
B(u, \varphi) & =B_{1}(u, \varphi)+B_{2}(u, \varphi)+B_{3}(u, \varphi), \\
B_{1}(u, \varphi) & =\sum_{j=1}^{n} \int_{\Omega} \mathcal{A}_{j}(x, u, \nabla u) D_{j} \varphi \omega d x=\int_{\Omega} \mathcal{A}(x, u, \nabla u) . \nabla \varphi \omega d x \\
B_{2}(u, \varphi) & =\int_{\Omega}|\Delta u|^{p-2} \Delta u \Delta \varphi \omega d x \\
B_{3}(u, \varphi) & =\int_{\Omega}|\Delta u|^{q-2} \Delta u \Delta \varphi \omega d x \\
T(\varphi) & =\int_{\Omega} f_{0}(x) \varphi(x) d x+\sum_{j=1}^{n} \int_{\Omega} f_{j}(x) D_{j} \varphi(x) d x .
\end{aligned}
$$

Then $u \in X$ is a (weak) solution to problem (P) if, for all $\varphi \in X$,

$$
B(u, \varphi)=B_{1}(u, \varphi)+B_{2}(u, \varphi)+B_{3}(u, \varphi)=T(\varphi) .
$$

Step 1. For $j=1, \ldots, n$ we define the operator $F_{j}: X \rightarrow L^{p^{\prime}}(\Omega, \omega)$ as

$$
\left(F_{j} u\right)(x)=\mathcal{A}_{j}(x, u(x), \nabla u(x)) .
$$

We now show that the operator $F_{j}$ is bounded and continuous.

(i) Using (H4), we obtain

$$
\begin{aligned}
\left\|F_{j} u\right\|_{L^{p^{p^{\prime}}(\Omega, \omega)}}^{p^{\prime}} & =\int_{\Omega}\left|F_{j} u(x)\right|^{p^{\prime}} \omega d x \\
& =\int_{\Omega}\left|\mathcal{A}_{j}(x, u, \nabla u)\right|^{p^{\prime}} \omega d x \\
& \leq \int_{\Omega}\left(K_{1}+h_{1}|u|^{p / p^{\prime}}+h_{2}|\nabla u|^{p / p^{\prime}}\right)^{p^{\prime}} \omega d x \\
& \leq C_{p} \int_{\Omega}\left[\left(K_{1}^{p^{\prime}}+h_{1}^{p^{\prime}}|u|^{p}+h_{2}^{p^{\prime}}|\nabla u|^{p}\right) \omega\right] d x \\
& =C_{p}\left[\int_{\Omega} K_{1}^{p^{\prime}} \omega d x+\int_{\Omega} h_{1}^{p^{\prime}}|u|^{p} \omega d x+\int_{\Omega} h_{2}^{p^{\prime}}|\nabla u|^{p} \omega d x\right],
\end{aligned}
$$

where the constant $C_{p}$ depends only on $p$. We have, by Theorem 3 (with $k=1$ ),

$$
\begin{aligned}
\int_{\Omega} h_{1}^{p^{\prime}}|u|^{p} \omega d x & \leq\left\|h_{1}\right\|_{L^{\infty}(\Omega)}^{p^{\prime}} \int_{\Omega}|u|^{p} \omega d x \\
& \leq C_{\Omega}^{p}\left\|h_{1}\right\|_{L^{\infty}(\Omega)}^{p^{\prime}} \int_{\Omega}|\nabla u|^{p} \omega d x \\
& \leq C_{\Omega}^{p}\left\|h_{1}\right\|_{L^{\infty}(\Omega)}^{p^{\prime}}\|u\|_{X^{\prime}}^{p}
\end{aligned}
$$

and

$$
\begin{aligned}
\int_{\Omega} h_{2}^{p^{\prime}}|\nabla u|^{p} \omega d x & \leq\left\|h_{2}\right\|_{L^{\infty}(\Omega)}^{p^{\prime}} \int_{\Omega}|\nabla u|^{p} \omega d x \\
& \leq\left\|h_{2}\right\|_{L^{\infty}(\Omega)}^{p^{\prime}}\|u\|_{X}^{p} .
\end{aligned}
$$

Therefore, in (3) we obtain

$$
\left\|F_{j} u\right\|_{L^{p^{\prime}}(\Omega, \omega)} \leq C_{p}^{1 / p^{\prime}}\left(\|K\|_{L^{p^{\prime}}(\Omega, \omega)}+\left(C_{\Omega}^{p / p^{\prime}}\left\|h_{1}\right\|_{L^{\infty}(\Omega)}+\left\|h_{2}\right\|_{L^{\infty}(\Omega)}\right)\|u\|_{X}^{p / p^{\prime}}\right) .
$$


(ii) Let $u_{m} \rightarrow u$ in $X$ as $m \rightarrow \infty$. We need to show that $F_{j} u_{m} \rightarrow F_{j} u$ in $L^{p^{\prime}}(\Omega, \omega)$. We will apply the Lebesgue Dominated Theorem. If $u_{m} \rightarrow u$ in $X$, then $\left|\nabla u_{m}\right| \rightarrow|\nabla u|$ in $L^{p}(\Omega, \omega)$. Using Theorem 2, there exist a subsequence $\left\{u_{m_{k}}\right\}$ and a function $\Phi_{1}$ in $L^{p}(\Omega, \omega)$ such that

$$
\begin{aligned}
& D_{j} u_{m_{k}}(x) \rightarrow D_{j} u(x), \text { a.e. in } \Omega, \\
& \left|\nabla u_{m_{k}}(x)\right| \leq \Phi_{1}(x), \text { a.e. in } \Omega .
\end{aligned}
$$

By Theorem 3 (with $k=1$ ),

$$
\left\|u_{m_{k}}\right\|_{L^{p}(\Omega, \omega)} \leq C_{\Omega}\left\|\left|\nabla u_{m_{k}}\right|\right\|_{L^{p}(\Omega, \omega)} \leq C_{\Omega}\left\|\Phi_{1}\right\|_{L^{p}(\Omega, \omega)}
$$

Next, applying (H4) we obtain

$$
\begin{aligned}
\left\|F_{j} u_{m_{k}}-F_{j} u\right\|_{L^{p^{\prime}(\Omega, \omega)}}^{p^{\prime}}= & \int_{\Omega}\left|F_{j} u_{m_{k}}(x)-F_{j} u(x)\right|^{p^{\prime}} \omega d x \\
= & \int_{\Omega}\left|\mathcal{A}_{j}\left(x, u_{m_{k}}, \nabla u_{m_{k}}\right)-\mathcal{A}_{j}(x, u, \nabla u)\right|^{p^{\prime}} \omega d x \\
\leq & C_{p} \int_{\Omega}\left(\left|\mathcal{A}_{j}\left(x, u_{m_{k}}, \nabla u_{m_{k}}\right)\right|^{p^{\prime}}+\left|\mathcal{A}_{j}(x, u, \nabla u)\right|^{p^{\prime}}\right) \omega d x \\
\leq & C_{p}\left[\int_{\Omega}\left(K_{1}+h_{1}\left|u_{m_{k}}\right|^{p / p^{\prime}}+h_{2}\left|\nabla u_{m_{k}}\right|^{p^{\prime} p^{\prime}}\right)^{p^{\prime}} \omega d x\right. \\
& \left.+\int_{\Omega}\left(K_{1}+h_{1}|u|^{p / p^{\prime}}+h_{2}|\nabla u|^{p / p^{\prime}}\right)^{p^{\prime}} \omega d x\right] \\
\leq & C_{p}\left[\int_{\Omega} K_{1}^{p^{\prime}} \omega d x+\left\|h_{1}\right\|_{L^{\infty}(\Omega)}^{p^{\prime}} \int_{\Omega}\left|u_{m_{k}}\right|^{p} \omega d x+\left\|h_{2}\right\|_{L^{\infty}(\Omega)}^{p^{\prime}(\Omega} \int_{\Omega}\left|\nabla u_{m_{k}}\right|^{p} \omega d x\right. \\
& \left.+\int_{\Omega} K_{1}^{p^{\prime}} \omega d x+\left\|h_{1}\right\|_{L^{\infty}(\Omega)}^{p^{\prime}} \int_{\Omega}|u|^{p} \omega d x+\left\|h_{2}\right\|_{L^{\infty}(\Omega)}^{p^{\prime}} \int_{\Omega}|\nabla u|^{p} \omega d x\right] \\
\leq & 2 C_{p}\left[\int_{\Omega} K_{1}^{p^{\prime}} \omega d x+\left\|h_{1}\right\|_{L^{\infty}(\Omega)}^{p^{\prime}} C_{\Omega}^{p} \int_{\Omega} \Phi_{1}^{p} \omega d x+\left\|h_{2}\right\|_{L^{\infty}(\Omega)}^{p^{\prime}} \int_{\Omega} \Phi_{1}^{p} \omega d x\right] \\
= & 2 C_{p}\left[\left\|K_{1}\right\|_{L^{p^{\prime}}(\Omega, \omega)}^{p^{\prime}}+\left(C_{\Omega}^{p}\left\|h_{1}\right\|_{L^{\infty}(\Omega)}^{p^{\prime}}+\left\|h_{2}\right\|_{L^{\infty}(\Omega)}^{p^{\prime}}\right)\left\|\Phi_{1}\right\|_{L^{p}(\Omega, \omega)}^{p}\right] .
\end{aligned}
$$

By condition (H1), we have

$$
F_{j} u_{m_{k}}(x)=\mathcal{A}_{j}\left(x, u_{m_{k}}(x), \nabla u_{m_{k}}(x)\right) \rightarrow \mathcal{A}_{j}(x, u(x), \nabla u(x))=F_{j} u(x),
$$

as $m_{k} \rightarrow+\infty$. Therefore, by the Lebesgue Dominated Convergence Theorem, we obtain

$$
\left\|F_{j} u_{m_{k}}-F_{j} u\right\|_{L^{p^{\prime}}(\Omega, \omega)} \rightarrow 0,
$$

that is, $F_{j} u_{m_{k}} \rightarrow F_{j} u$ in $L^{p^{\prime}}(\Omega, \omega)$. We conclude from the Convergence Principle in Banach spaces (see Proposition 10.13 in [22]) that

$$
F_{j} u_{m} \rightarrow F_{j} u \text { in } L^{p^{\prime}}(\Omega, \omega) .
$$

Step 2. We define the operator $G_{1}: X \rightarrow L^{p^{\prime}}(\Omega, \omega)$ by

$$
\left(G_{1} u\right)(x)=|\Delta u(x)|^{p-2} \Delta u(x) .
$$

This operator is continuous and bounded. In fact, 
(i) We have

$$
\begin{aligned}
\left\|G_{1} u\right\|_{L^{p^{\prime}}(\Omega, \omega)}^{p^{\prime}} & =\left.\left.\int_{\Omega}|| \Delta u\right|^{p-2} \Delta u\right|^{p^{\prime}} \omega d x \\
& =\int_{\Omega}|\Delta u|^{(p-1) p^{\prime}} \omega d x \\
& =\int_{\Omega}|\Delta u|^{p} \omega d x \\
& \leq\|u\|_{X}^{p} .
\end{aligned}
$$

Hence, $\left\|G_{1} u\right\|_{L^{p^{\prime}}(\Omega, \omega)} \leq\|u\|_{X}^{p / p^{\prime}}$.

(ii) If $u_{m} \rightarrow u$ in $X$ then $\Delta u_{m} \rightarrow \Delta u$ in $L^{p}(\Omega, \omega)$. By Theorem 2, there exist a subsequence $\left\{u_{m_{k}}\right\}$ and a function $\Phi_{2} \in L^{p}(\Omega, \omega)$ such that

$$
\begin{aligned}
& \Delta u_{m_{k}}(x) \rightarrow \Delta u(x), \text { a.e. in } \Omega, \\
& \left|\Delta u_{m_{k}}(x)\right| \leq \Phi_{2}(x), \text { a.e. in } \Omega .
\end{aligned}
$$

Hence, using Lemma 1(a), we obtain (since $2 \leq q<p<\infty)$ with $\theta=p / p^{\prime}=p-1$ and $\theta^{\prime}=(p-$ $1) /(p-2)$,

$$
\begin{aligned}
& \left\|G_{1} u_{m_{k}}-G_{1} u\right\|_{L^{p^{\prime}}(\Omega, \omega)}^{p^{\prime}}=\int_{\Omega}\left|G_{1} u_{m_{k}}-G_{1} u\right|^{p^{\prime}} \omega d x \\
& =\left.\int_{\Omega}|| \Delta u_{m_{k}}\right|^{p-2} \Delta u_{m_{k}}-\left.|\Delta u|^{p-2} \Delta u\right|^{p^{\prime}} \omega d x \\
& \leq \int_{\Omega}\left[\alpha_{p}\left|\Delta u_{m_{k}}-\Delta u\right|\left(\left|\Delta u_{m_{k}}\right|+|\Delta u|\right)^{(p-2)}\right]^{p^{\prime}} \omega d x \\
& \leq \alpha_{p}^{p^{\prime}} \int_{\Omega}\left|\Delta u_{m_{k}}-\Delta u\right|^{p^{\prime}}\left(2 \Phi_{2}\right)^{(p-2) p^{\prime}} \omega d x \\
& \leq 2^{(p-2) p^{\prime}} \alpha_{p}^{p^{\prime}}\left(\int_{\Omega}\left|\Delta u_{m_{k}}-\Delta u\right|^{p^{\prime} \theta} \omega d x\right)^{1 / \theta}\left(\int_{\Omega} \Phi_{2}^{(p-2) p^{\prime} \theta^{\prime}} \omega d x\right)^{1 / \theta^{\prime}} \\
& \leq \alpha_{p}^{p^{\prime}} 2^{(p-2) p^{\prime}}\left(\int_{\Omega}\left|\Delta u_{m_{k}}-\Delta u\right|^{p} \omega d x\right)^{p^{\prime} / p}\left(\int_{\Omega} \Phi_{2}^{p} \omega d x\right)^{(p-2) /(p-1)} \\
& \leq \alpha_{p}^{p^{\prime}} 2^{(p-2) p^{\prime}}\left\|u_{m_{k}}-u\right\|_{X}^{p^{\prime}}\left\|\Phi_{2}\right\|_{L^{p}(\Omega, \omega)}^{(p-2) p^{\prime}}
\end{aligned}
$$

since $(p-2) p^{\prime} \theta^{\prime}=(p-2) \frac{p}{(p-1)} \frac{(p-1)}{(p-2)}=p$ if $p \neq 2$. Hence

$$
\left\|G_{1} u_{m_{k}}-G_{1} u\right\|_{L^{p^{\prime}(\Omega, \omega)}} \leq 2^{p-2} \alpha_{p}\left\|\Phi_{2}\right\|_{L^{p}(\Omega, \omega)}^{p-2}\left\|u_{m_{k}}-u\right\|_{X} .
$$

Therefore, by the Lebesgue Dominated Convergence Theorem, we obtain (when $m_{k} \rightarrow \infty$ )

$$
\left\|G_{1} u_{m_{k}}-G_{1} u\right\|_{X} \rightarrow 0
$$

that is, $G_{1} u_{m_{k}} \rightarrow G_{1} u$ in $L^{p^{\prime}}(\Omega, \omega)$. By the Convergence Principle in Banach spaces (see Proposition 10.13 in [22]), we have

$$
G_{1} u_{m} \rightarrow G_{1} u \text { in } L^{p^{\prime}}(\Omega, \omega) .
$$

Step 3. We define the operator $G_{2}: X \rightarrow L^{p^{\prime}}(\Omega, \omega)$ by

$$
\left(G_{2} u\right)(x)=|\Delta u(x)|^{q-2} \Delta u(x) .
$$

We also have that the operator $G_{2}$ is continuous and bounded. In fact, 
(i) We have, with $r=(p-1) /(q-1)>1$ and $r^{\prime}=(p-1) /(p-q)$, that

$$
\begin{aligned}
\left\|G_{2} u\right\|_{L^{p^{\prime}(\Omega, \omega)}}^{p^{\prime}} & =\int_{\Omega} \|\left.\left.\Delta u\right|^{q-2} \Delta u\right|^{p^{\prime}} \omega d x \\
& =\int_{\Omega}|\Delta u|^{(q-1) p^{\prime}} \omega d x \\
& \leq\left(\int_{\Omega}|\Delta u|^{(q-1) p^{\prime} r} \omega d x\right)^{1 / r}\left(\int_{\Omega} \omega d x\right)^{1 / r^{\prime}} \\
& =\left(\int_{\Omega}|\Delta u|^{p} \omega d x\right)^{(q-1) /(p-1)}\left(\int_{\Omega} \omega d x\right)^{(p-q) /(p-1)} \\
& =C_{p, q}\|\Delta u\|_{L^{p}(\Omega, \omega)}^{(q-1) p^{\prime}} \\
& \leq C_{p, q}\|u\|_{X}^{(q-1) p^{\prime}},
\end{aligned}
$$

where $C_{p, q}=\left(\int_{\Omega} \omega d x\right)^{(p-q) /(p-1)}$. Hence $\left\|G_{2} u\right\|_{L^{p^{\prime}}(\Omega, \omega)} \leq C_{p, q}^{1 / p^{\prime}}\|u\|_{X}^{q-1}$.

(ii) If $u_{m} \rightarrow u$ in $X$ then $\Delta u_{m} \rightarrow \Delta u$ in $L^{p}(\Omega, \omega)$. If $2<q<p<\infty$, by (5), (6) and Lemma 1(a), we have

$$
\begin{aligned}
\left\|G_{2} u_{m_{k}}-G_{2} u\right\|_{L^{p^{\prime}(\Omega, \omega)}}^{p^{\prime}} & =\left.\int_{\Omega}|| \Delta u_{m_{k}}\right|^{q-2} \Delta u_{m_{k}}-\left.|\Delta u|^{q-2} \Delta u\right|^{p^{\prime}} \omega d x \\
& \leq \int_{\Omega}\left[\alpha_{q}\left|\Delta u_{m_{k}}-\Delta u\right|\left(\left|\Delta u_{m_{k}}\right|+|\Delta u|\right)^{q-2}\right]^{p^{\prime}} \omega d x \\
& =\alpha_{q}^{p^{\prime}} \int_{\Omega}\left|\Delta u_{m_{k}}-\Delta u\right|^{p^{\prime}}\left(\left|\Delta u_{m_{k}}\right|+|\Delta u|\right)^{(q-2) p^{\prime}} \omega d x \\
& \leq 2^{(q-2) p^{\prime}} \alpha_{q}^{p^{\prime}} \int_{\Omega}\left|\Delta u_{m_{k}}-\Delta u\right|^{p^{\prime}} \Phi_{2}^{(q-2) p^{\prime}} \omega d x
\end{aligned}
$$

For $s=p / p^{\prime}=p-1>1$ and $s^{\prime}=(p-1) /(p-2)$, we have in (8)

$$
\begin{aligned}
& \left\|G_{2} u_{m_{k}}-G_{2} u\right\|_{L^{p^{\prime}}(\Omega, \omega)}^{p^{\prime}} \\
& \leq 2^{(q-2) p^{\prime}} \alpha_{q}^{p^{\prime}} \int_{\Omega}\left|\Delta u_{m_{k}}-\Delta u\right|^{p^{\prime}} \Phi_{2}^{(q-2) p^{\prime}} \omega d x \\
& \leq 2^{(q-2) p^{\prime}} \alpha_{q}^{p^{\prime}}\left(\int_{\Omega}\left|\Delta u_{m_{k}}-\Delta u\right|^{p^{\prime} s} \omega d x\right)^{1 / s}\left(\int_{\Omega} \Phi_{2}^{(q-2) p^{\prime} s^{\prime}} \omega d x\right)^{1 / s^{\prime}} \\
& =2^{(q-2) p^{\prime}} \alpha_{q}^{p^{\prime}}\left(\int_{\Omega}\left|\Delta u_{m_{k}}-\Delta u\right|^{p} \omega d x\right)^{p^{\prime / p}}\left(\int_{\Omega} \Phi_{2}^{(q-2) p /(p-2)} \omega d x\right)^{(p-2) /(p-1)} .
\end{aligned}
$$

Now, since $0<\eta=\frac{(q-2) p}{(p-2)}<p$, then by Remark 1, we have $\left\|\Phi_{2}\right\|_{L^{\eta}(\Omega, \omega)} \leq C_{p, \eta}\left\|\Phi_{2}\right\|_{L^{p}(\Omega, \omega)}$. Therefore, we obtain

$$
\left\|G_{2} u_{m_{k}}-G_{2} u\right\|_{L^{p^{\prime}}(\Omega, \omega)}^{p^{\prime}} \leq 2^{(q-2) p^{\prime}} \alpha_{q}^{p^{\prime}} C_{p, \eta}^{(q-2) p^{\prime}}\left\|u_{m_{k}}-u\right\|_{X}^{p^{\prime}}\left\|\Phi_{2}\right\|_{L^{p}(\Omega, \omega)}^{(q-2) p^{\prime}} .
$$

Hence $\left\|G_{2} u_{m_{k}}-G_{2} u\right\|_{L^{p^{\prime}}(\Omega, \omega)} \leq 2^{q-2} \alpha_{q} C_{p, \eta}^{q-2}\left\|\Phi_{2}\right\|_{L^{p}(\Omega, \omega)}^{q-2}\left\|u_{m_{k}}-u\right\|_{X}$.

In the case $2=q<p<\infty$, we have $\left(G_{2} u\right)(x)=\Delta u(x)$ and

$$
\begin{aligned}
\left\|G_{2} u_{m_{k}}-G_{2} u\right\|_{L^{p^{\prime}(\Omega, \omega)}}^{p^{\prime}} & =\int_{\Omega}\left|\Delta u_{m_{k}}-\Delta u\right|^{p^{\prime}} \omega d x \\
& \leq\left(\int_{\Omega}\left|\Delta u_{m_{k}}-\Delta u\right|^{p} \omega d x\right)^{p^{\prime} / p}\left(\int_{\Omega} \omega d x\right)^{(p-2) /(p-1)} \\
& \leq\left\|u_{m_{k}}-u\right\|_{X}^{p^{\prime}}\left(\int_{\Omega} \omega d x\right)^{(p-2) /(p-1)} .
\end{aligned}
$$


Therefore, for $2 \leq q<p<\infty$, by the Dominated Convergence Theorem we obtain (when $m_{k} \rightarrow \infty$ )

$$
\left\|G_{2} u_{m_{k}}-G_{2} u\right\|_{L^{p^{\prime}}(\Omega, \omega)} \rightarrow 0
$$

that is, $G_{2} u_{m_{k}} \rightarrow G_{2} u$ in $L^{p^{\prime}}(\Omega, \omega)$. By the Convergence Principle in Banach spaces, we have

$$
G_{2} u_{m} \rightarrow G_{2} u \text { in } L^{p^{\prime}}(\Omega, \omega)
$$

Step 4. Since $\frac{f_{j}}{\omega} \in L^{p^{\prime}}(\Omega, \omega)(j=0,1, \ldots, n)$, then $T \in\left[W_{0}^{1 . p}(\Omega, \omega)\right]^{*} \subset X^{*}$. Moreover, we have by Theorem 3 (with $k=1$ ),

$$
\begin{aligned}
|T(\varphi)| & \leq \int_{\Omega}\left|f_{0}\right||\varphi| d x+\sum_{j=1}^{n} \int_{\Omega}\left|f_{j}\right|\left|D_{j} \varphi\right| d x \\
& =\int_{\Omega} \frac{\left|f_{0}\right|}{\omega}|\varphi| \omega d x+\sum_{j=1}^{n} \int_{\Omega} \frac{\left|f_{j}\right|}{\omega}\left|D_{j} \varphi\right| \omega d x \\
& \leq\left\|f_{0} / \omega\right\|_{L^{p^{\prime}}(\Omega, \omega)}\|\varphi\|_{L^{p}(\Omega, \omega)}+\sum_{j=1}^{n}\left\|f_{j} / \omega\right\|_{L^{p^{\prime}}(\Omega, \omega)}\left\|D_{j} \varphi\right\|_{L^{p}(\Omega, \omega)} \\
& \leq C_{\Omega}\left\|f_{0} / \omega\right\|_{L^{p^{\prime}}(\Omega, \omega)}\|\nabla \varphi\|_{L^{p}(\Omega, \omega)}+\left(\sum_{j=1}^{n}\left\|f_{j} / \omega\right\|_{L^{p^{\prime}}(\Omega, \omega)}\right)\|\nabla \varphi\|_{L^{p}(\Omega, \omega)} \\
& \leq\left(C_{\Omega}\left\|f_{0} / \omega\right\|_{L^{p^{\prime}}(\Omega, \omega)}+\sum_{j=1}^{n}\left\|f_{j} / \omega\right\|_{L^{p^{\prime}}(\Omega, \omega)}\right)\|\varphi\|_{X} \cdot
\end{aligned}
$$

Moreover, we also have

$$
\begin{aligned}
|B(u, \varphi)| & \leq\left|B_{1}(u, \varphi)\right|+\left|B_{2}(u, \varphi)\right|+\left|B_{3}(u, \varphi)\right| \\
& \leq \sum_{j=1}^{n} \int_{\Omega}\left|\mathcal{A}_{j}(x, u, \nabla u)\right|\left|D_{j} \varphi\right| \omega d x+\int_{\Omega}|\Delta u|^{p-2}|\Delta u||\Delta \varphi| \omega d x+\int_{\Omega}|\Delta u|^{q-2}|\Delta u||\Delta \varphi| \omega d x
\end{aligned}
$$

In (10) we have, using (H4), we have

$$
\begin{aligned}
& \int_{\Omega}|\mathcal{A}(x, u, \nabla u)||\nabla \varphi| \omega d x \leq \int_{\Omega}\left(K_{1}+h_{1}|u|^{p / p^{\prime}}+h_{2}|\nabla u|^{p / p^{\prime}}\right)|\nabla \varphi| \omega d x \\
& \leq\left\|K_{1}\right\|_{L^{p^{\prime}(\Omega, \omega)}}\||\nabla \varphi|\|_{L^{p}(\Omega, \omega)}+\left\|h_{1}\right\|_{L^{\infty}(\Omega)}\|u\|_{L^{p}(\Omega, \omega)}^{p / p^{\prime}}\||\nabla \varphi|\|_{L^{p}(\Omega, \omega)} \\
& +\left\|h_{2}\right\|_{L^{\infty}(\Omega)}\||\nabla u|\|_{L^{p}(\Omega, \omega)}^{p / p^{\prime}}\||\nabla \varphi|\|_{L^{p}(\Omega, \omega)} \\
& \leq\left(\left\|K_{1}\right\|_{L^{p^{\prime}(\Omega, \omega)}}+\left(C_{\Omega}^{p / p^{\prime}}\left\|h_{1}\right\|_{L^{\infty}(\Omega)}+\left\|h_{2}\right\|_{L^{\infty}(\Omega)}\right)\|u\|_{X}^{p / p^{\prime}}\right)\|\varphi\|_{X^{\prime}}
\end{aligned}
$$

and

$$
\begin{aligned}
\int_{\Omega}|\Delta u|^{p-2}|\Delta u||\Delta \varphi| \omega d x & =\int_{\Omega}|\Delta u|^{p-1}|\Delta \varphi| \omega d x \\
& \leq\left(\int_{\Omega}|\Delta u|^{p} \omega d x\right)^{1 / p^{\prime}}\left(\int_{\Omega}|\Delta \varphi|^{p} \omega d x\right)^{1 / p} \\
& \leq\|u\|_{X}^{p / p^{\prime}}\|\varphi\|_{X^{\prime}}
\end{aligned}
$$

and

$$
\begin{aligned}
\int_{\Omega}|\Delta u|^{q-2}|\Delta u||\Delta \varphi| \omega d x & =\int_{\Omega}|\Delta u|^{q-1}|\Delta \varphi| \omega d x \\
& \leq\left(\int_{\Omega}|\Delta u|^{(q-1) p^{\prime}} \omega d x\right)^{1 / p^{\prime}}\left(\int_{\Omega}|\Delta \varphi|^{p} \omega d x\right)^{1 / p} \\
& =\|\Delta u\|_{L^{p^{\prime}(q-1)}(\Omega, \omega)}^{q-1}\|\Delta \varphi\|_{L^{p}(\Omega, \omega)}
\end{aligned}
$$


Since $0<\eta=p^{\prime}(q-1)=\frac{p(q-1)}{p-1}<p$, then by Remark 1 we have

$$
\|\Delta u\|_{L^{\eta}(\Omega, \omega)} \leq C_{p, \eta}\|\Delta u\|_{L^{p}(\Omega, \omega)} .
$$

Hence

$$
\begin{aligned}
\int_{\Omega}|\Delta u|^{q-2}|\Delta u \| \Delta \varphi| \omega d x & \leq\|\Delta u\|_{L^{p^{\prime}(q-1)}(\Omega, \omega)}^{q-1}\|\Delta \varphi\|_{L^{p}(\Omega, \omega)} \\
& \leq C_{p, \eta}^{q-1}\|\Delta u\|_{L^{p}(\Omega, \omega)}^{q-1}\|\Delta \varphi\|_{L^{p}(\Omega, \omega)} \\
& \leq C_{p, \eta}^{q-1}\|u\|_{X}^{q-1}\|\varphi\|_{X} .
\end{aligned}
$$

Hence, in (10) we obtain, for all $u, \varphi \in X$

$|B(u, \varphi)| \leq\left[\left\|K_{1}\right\|_{L^{p^{\prime}}(\Omega, \omega)}+C_{\Omega}^{p / p^{\prime}}\left\|h_{1}\right\|_{L^{\infty}(\Omega)}\|u\|_{X}^{p / p^{\prime}}+\left\|h_{2}\right\|_{L^{\infty}(\Omega, \omega)}\|u\|_{X}^{p / p^{\prime}}+\|u\|_{X}^{p / p^{\prime}}+C_{p, \eta}^{q-1}\|u\|_{X}^{q-1}\right]\|\varphi\|_{X}$.

Since $B(u,$.$) is linear, for each u \in X$, there exists a linear and continuous functional on $X$ denoted by $A u$ such that $\langle A u, \varphi\rangle=B(u, \varphi)$, for all $u, \varphi \in X$ (here $\langle f, x\rangle$ denotes the value of the linear functional $f$ at the point $x$ ). Moreover

$$
\|A u\|_{*} \leq\left\|K_{1}\right\|_{L^{p^{\prime}}(\Omega, \omega)}+C_{\Omega}^{p / p^{\prime}}\left\|h_{1}\right\|_{L^{\infty}(\Omega)}\|u\|_{X}^{p / p^{\prime}}+\left\|h_{2}\right\|_{L^{\infty}(\Omega, \omega)}\|u\|_{X}^{p / p^{\prime}}+\|u\|_{X}^{p / p^{\prime}}+C_{p, \eta}^{q-1}\|u\|_{X}^{q-1},
$$

where $\|A u\|_{*}=\sup \left\{|\langle A u, \varphi\rangle|=|B(u, \varphi)|: \varphi \in X,\|\varphi\|_{X}=1\right\}$ is the norm of the operators $A u$. Hence, we obtain the operator $A: X \rightarrow X^{*}, u \mapsto A u$. Consequently, Problem (P) is equivalent to the operator equation

$$
A u=T, u \in X
$$

Step 5. Using condition (H2) and Lemma 1(b), we have

$$
\begin{aligned}
& \left\langle A u_{1}-A u_{2}, u_{1}-u_{2}\right\rangle=B\left(u_{1}, u_{1}-u_{2}\right)-B\left(u_{2}, u_{1}-u_{2}\right) \\
& =\int_{\Omega} \mathcal{A}\left(x, u_{1}, \nabla u_{1}\right) \cdot \nabla\left(u_{1}-u_{2}\right) \omega d x+\int_{\Omega}\left|\Delta u_{1}\right|^{p-2} \Delta u_{1} \Delta\left(u_{1}-u_{2}\right) \omega d x \\
& +\int_{\Omega}\left|\Delta u_{1}\right|^{q-2} \Delta u_{1} \Delta\left(u_{1}-u_{2}\right) \omega d x-\int_{\Omega} \mathcal{A}\left(x, u_{2}, \nabla u_{2}\right) \cdot \nabla\left(u_{1}-u_{2}\right) \omega d x \\
& -\int_{\Omega}\left|\Delta u_{2}\right|^{p-2} \Delta u_{2} \Delta\left(u_{1}-u_{2}\right) \omega d x-\int_{\Omega}\left|\Delta u_{2}\right|^{q-2} \Delta u_{2} \Delta\left(u_{1}-u_{2}\right) \omega d x \\
& =\int_{\Omega}\left(\mathcal{A}\left(x, u_{1}, \nabla u_{1}\right)-\mathcal{A}\left(x, u_{2}, \nabla u_{2}\right)\right) \cdot \nabla\left(u_{1}-u_{2}\right) \omega d x \\
& +\int_{\Omega}\left(\left|\Delta u_{1}\right|^{p-2} \Delta u_{1}-\left|\Delta u_{2}\right|^{p-2} \Delta u_{2}\right) \Delta\left(u_{1}-u_{2}\right) \omega d x \\
& +\int_{\Omega}\left(\left|\Delta u_{1}\right|^{q-2} \Delta u_{1}-\left|\Delta u_{2}\right|^{q-2} \Delta u_{2}\right) \Delta\left(u_{1}-u_{2}\right) \omega d x \\
& \geq \theta_{1} \int_{\Omega}\left|\nabla\left(u_{1}-u_{2}\right)\right|^{p} \omega d x+\beta_{p} \int_{\Omega}\left(\left|\Delta u_{1}\right|+\left|\Delta u_{2}\right|\right)^{p-2}\left|\Delta u_{1}-\Delta u_{2}\right|^{2} \omega d x \\
& +\beta_{q} \int_{\Omega}\left(\left|\Delta u_{1}\right|+\left|\Delta u_{2}\right|\right)^{q-2}\left|\Delta u_{1}-\Delta u_{2}\right|^{2} \omega d x \\
& \geq \theta_{1} \int_{\Omega}\left|\nabla\left(u_{1}-u_{2}\right)\right|^{p} \omega d x+\beta_{p} \int_{\Omega}\left(\left|\Delta u_{1}-\Delta u_{2}\right|\right)^{p-2}\left|\Delta u_{1}-\Delta u_{2}\right|^{2} \omega d x \\
& +\beta_{q} \int_{\Omega}\left(\left|\Delta u_{1}-\Delta u_{2}\right|\right)^{q-2}\left|\Delta u_{1}-\Delta u_{2}\right|^{2} \omega d x \\
& =\theta_{1} \int_{\Omega}\left|\nabla\left(u_{1}-u_{2}\right)\right|^{p} \omega d x+\beta_{p} \int_{\Omega}\left|\Delta u_{1}-\Delta u_{2}\right|^{p} \omega d x+\beta_{q} \int_{\Omega}\left|\Delta u_{1}-\Delta u_{2}\right|^{q} \omega d x \\
& \geq \theta_{1} \int_{\Omega}\left|\nabla\left(u_{1}-u_{2}\right)\right|^{p} \omega d x+\beta_{p} \int_{\Omega}\left|\Delta u_{1}-\Delta u_{2}\right|^{p} \omega d x \\
& \geq \theta\left\|u_{1}-u_{2} \mid\right\|_{X}^{p}
\end{aligned}
$$


where $\theta=\min \left\{\theta_{1}, \beta_{p}\right\}$. Therefore, the operator $A$ is strongly monotone, and this implies that $A$ is strictly monotone. Moreover, from (H3), we obtain

$$
\begin{aligned}
\langle A u, u\rangle & =B(u, u)=B_{1}(u, u)+B_{2}(u, u)+B_{3}(u, u) \\
& =\int_{\Omega} \mathcal{A}(x, u, \nabla u) . \nabla u \omega d x+\int_{\Omega}|\Delta u|^{p-2} \Delta u \Delta u \omega d x+\int_{\Omega}|\Delta u|^{q-2} \Delta u \Delta u \omega d x \\
& \geq \int_{\Omega} \lambda_{1}|\nabla u|^{p} \omega d x+\int_{\Omega}|\Delta u|^{p} \omega d x+\int_{\Omega}|\Delta u|^{q} \omega d x \\
& \geq \int_{\Omega} \lambda_{1}|\nabla u|^{p} \omega d x+\int_{\Omega}|\Delta u|^{p} \omega d x \\
& \geq \gamma\|u\|_{X^{\prime}}^{p}
\end{aligned}
$$

where $\gamma=\min \left\{\lambda_{1}, 1\right\}$. Hence, since $2 \leq q<p<\infty$, we have

$$
\frac{\langle A u, u\rangle}{\|u\|_{X}} \rightarrow+\infty, \text { as }\|u\|_{X} \rightarrow+\infty
$$

that is, $A$ is coercive.

Step 6. We need to show that the operator $A$ is continuous. Let $u_{m} \rightarrow u$ in $X$ as $m \rightarrow \infty$. We have

$$
\begin{aligned}
\left|B_{1}\left(u_{m}, \varphi\right)-B_{1}(u, \varphi)\right| & \leq \sum_{j=1}^{n} \int_{\Omega}\left|\mathcal{A}_{j}\left(x, u_{m}, \nabla u_{m}\right)-\mathcal{A}_{j}(x, u, \nabla u)\right|\left|D_{j} \varphi\right| \omega d x \\
& =\sum_{j=1}^{n} \int_{\Omega}\left|F_{j} u_{m}-F_{j} u \| D_{j} \varphi\right| \omega d x \\
& \leq \sum_{j=1}^{n}\left\|F_{j} u_{m}-F_{j} u\right\|_{L^{p^{\prime}}(\Omega, \omega)}\left\|D_{j} \varphi\right\|_{L^{p}(\Omega, \omega)} \\
& \leq\left(\sum_{j=1}^{n}\left\|F_{j} u_{m}-F_{j} u\right\|_{L^{p^{\prime}}(\Omega, \omega)}\right)\|\varphi\|_{X^{\prime}}
\end{aligned}
$$

and

$$
\begin{aligned}
\left|B_{2}\left(u_{m}, \varphi\right)-B_{2}(u, \varphi)\right| & =\left.\left|\int_{\Omega}\right| \Delta u_{m}\right|^{p-2} \Delta u_{m} \Delta \varphi \omega d x-\int_{\Omega}|\Delta u|^{p-2} \Delta u \Delta \varphi \omega d x \mid \\
& \leq\left.\int_{\Omega}|| \Delta u_{m}\right|^{p-2} \Delta u_{m}-|\Delta u|^{p-2} \Delta u|| \Delta \varphi \mid \omega d x \\
& =\int_{\Omega}\left|G_{1} u_{m}-G_{1} u\right||\Delta \varphi| \omega d x \\
& \leq\left\|G_{1} u_{m}-G_{1} u\right\|_{L^{p^{\prime}(\Omega, \omega)}}\|\Delta \varphi\|_{L^{p}(\Omega, \omega)} \\
& \leq\left\|G_{1} u_{m}-G_{1} u\right\|_{L^{p^{\prime}(\Omega, \omega)}}\|\varphi\|_{X} .
\end{aligned}
$$

and

$$
\begin{aligned}
\left|B_{3}\left(u_{m}, \varphi\right)-B_{3}(u, \varphi)\right| & =\left.\left|\int_{\Omega}\right| \Delta u_{m}\right|^{q-2} \Delta u_{m} \Delta \varphi \omega d x-\int_{\Omega}|\Delta u|^{q-2} \Delta u \Delta \varphi \omega d x \mid \\
& \leq\left.\int_{\Omega}|| \Delta u_{m}\right|^{q-2} \Delta u_{m}-|\Delta u|^{q-2} \Delta u|| \Delta \varphi \mid \omega d x \\
& =\int_{\Omega}\left|G_{2} u_{m}-G_{2} u\right||\Delta \varphi| \omega d x \\
& \leq\left\|G_{2} u_{m}-G_{2} u\right\|_{L^{p^{\prime}}(\Omega, \omega)}\|\varphi\|_{X},
\end{aligned}
$$

for all $\varphi \in X$. Hence

$$
\begin{aligned}
\left|B\left(u_{m}, \varphi\right)-B(u, \varphi)\right| & \leq\left|B_{1}\left(u_{m}, \varphi\right)-B_{1}(u, \varphi)\right|+\left|B_{2}\left(u_{m}, \varphi\right)-B_{2}(u, \varphi)\right|+\left|B_{3}\left(u_{m}, \varphi\right)-B_{3}(u, \varphi)\right| \\
& \leq\left[\sum_{j=1}^{n}\left\|F_{j} u_{m}-F_{j} u\right\|_{L^{p^{\prime}}(\Omega, \omega)}+\left\|G_{1} u_{m}-G_{1} u\right\|_{L^{p^{\prime}}(\Omega, \omega)}+\left\|G_{2} u_{m}-G_{2} u\right\|_{L^{p^{\prime}}(\Omega, \omega)}\right]\|\varphi\|_{X} .
\end{aligned}
$$


Then we obtain

$$
\left\|A u_{m}-A u\right\|_{*} \leq \sum_{j=1}^{n}\left\|F_{j} u_{m}-F_{j} u\right\|_{L^{p^{\prime}}(\Omega, \omega)}+\left\|G_{1} u_{m}-G_{1} u\right\|_{L^{p^{\prime}}(\Omega, \omega)}+\left\|G_{2} u_{m}-G_{2} u\right\|_{L^{p^{\prime}}(\Omega, \omega)} .
$$

Therefore, using (4), (7) and (9) we have $\left\|A u_{m}-A u\right\|_{*} \rightarrow 0$ as $m \rightarrow+\infty$, that is, $A$ is continuous and this implies that $A$ is hemicontinuous.

Therefore, by Theorem 4, the operator equation $A u=T$ has a unique solution $u \in X$ and it is the unique solution for problem $(\mathrm{P})$.

Step 7. In particular, by setting $\varphi=u$ in Definition 4, we have

$$
B(u, u)=B_{1}(u, u)+B_{2}(u, u)+B_{3}(u, u)=T(u) .
$$

Hence, using (H3) and $\gamma=\min \left\{\lambda_{1}, 1\right\}$, we obtain

$$
\begin{aligned}
& B_{1}(u, u)+B_{2}(u, u)+B_{3}(u, u) \\
& =\int_{\Omega} \mathcal{A}(x, u, \nabla u) . \nabla u \omega d x+\int_{\Omega}|\Delta u|^{p-2} \Delta u \Delta u \omega d x+\int_{\Omega}|\Delta u|^{q-2} \Delta u \Delta u \omega d x \\
& \geq \int_{\Omega} \lambda_{1}|\nabla u|^{p}+\int_{\Omega}|\Delta u|^{p} \omega d x+\int_{\Omega}|\Delta u|^{q} \omega d x \\
& \geq \int_{\Omega} \lambda_{1}|\nabla u|^{p}+\int_{\Omega}|\Delta u|^{p} \omega d x \\
& \geq \gamma\|u\|_{X}^{p}
\end{aligned}
$$

and

$$
\begin{aligned}
T(u) & =\int_{\Omega} f_{0} u d x+\sum_{j=1}^{n} \int_{\Omega} f_{j} D_{j} u d x \\
& \leq\left\|f_{0} / \omega\right\|_{L^{p^{\prime}}(\Omega, \omega)}\|u\|_{L^{p}(\Omega, \omega)}+\sum_{j=1}^{n}\left\|f_{j} /\left.\omega\right|_{L^{p^{\prime}(\Omega)}}\right\| D_{j} u \|_{L^{p}(\Omega, \omega)} \\
& \leq\left(C_{\Omega}\left\|f_{0} / \omega\right\|_{L^{p^{\prime}}(\Omega, \omega)}+\sum_{j=1}^{n}\left\|f_{j} / \omega\right\|_{L^{p^{\prime}}(\Omega)}\right)\|u\|_{X} .
\end{aligned}
$$

Therefore, in (11), we obtain

$$
\gamma\|u\|_{X}^{p} \leq\left(C_{\Omega}\left\|f_{0} / \omega\right\|_{L^{p^{\prime}}(\Omega, \omega)}+\sum_{j=1}^{n}\left\|f_{j} / \omega\right\|_{L^{p^{\prime}}(\Omega, \omega)}\right)\|u\|_{X}
$$

and we obtain

$$
\|u\|_{X} \leq \frac{1}{\gamma^{p^{\prime} / p}}\left(C_{\Omega}\left\|f_{0} / \omega\right\|_{L^{p^{\prime}}(\Omega, \omega)}+\sum_{j=1}^{n}\left\|f_{j} / \omega\right\|_{L^{p^{\prime}}(\Omega, \omega)}\right)^{p^{\prime} / p} .
$$

Example 1. Let $\Omega=\left\{(x, y) \in \mathbb{R}^{2}: x^{2}+y^{2}<1\right\}$, the weight function $\omega(x, y)=\left(x^{2}+y^{2}\right)^{-1 / 2}\left(\omega \in A_{4}, p=4\right.$ and $q=3)$, and the function

$$
\begin{aligned}
& \mathcal{A}: \Omega \times \mathbb{R} \times \mathbb{R}^{2} \rightarrow \mathbb{R}^{2} \text { defined by } \\
& \mathcal{A}((x, y), \eta, \xi)=h_{2}(x, y)|\xi| \xi,
\end{aligned}
$$

where $h(x, y)=2 e^{\left(x^{2}+y^{2}\right)}$. Let us consider the partial differential operator

$$
L u(x, y)=\Delta\left[\left(x^{2}+y^{2}\right)^{-1 / 2}\left(|\Delta u|^{2} \Delta u+|\Delta u| \Delta u\right)\right]-\operatorname{div}\left(\left(x^{2}+y^{2}\right)^{-1 / 2} \mathcal{A}((x, y), u, \nabla u)\right) .
$$

Therefore, by Theorem 1, the problem 


$$
\begin{cases}L u(x)=\frac{\cos (x y)}{\left(x^{2}+y^{2}\right)}-\frac{\partial}{\partial x}\left(\frac{\sin (x y)}{\left(x^{2}+y^{2}\right)}\right)-\frac{\partial}{\partial y}\left(\frac{\sin (x y)}{\left(x^{2}+y^{2}\right)}\right), & \text { in } \Omega \\ u(x)=0, & \text { on } \partial \Omega\end{cases}
$$

has a unique solution $u \in X=W^{2,4}(\Omega, \omega) \cap W_{0}^{1,4}(\Omega, \omega)$.

Conflicts of Interest: “The author declares no conflict of interest."

\section{References}

[1] Andersson, L. E., Elfving, T., \& Golub, G. H. (1998). Solution of biharmonic equations with application to radar imaging. Journal of Computational and Applied Mathematics, 94(2), 153-180.

[2] Lai, M. C., \& Liu, H. C. (2005). Fast direct solver for the biharmonic equation on a disk and its application to incompressible flows. Applied Mathematics and Computation, 164(3), 679-695.

[3] Cavalheiro, A. C. (2013). Existence and uniqueness of solutions for some degenerate nonlinear Dirichlet problems. Journal of Applied Analysis, 19(1), 41-54.

[4] Cavalheiro, A. C. (2013). Existence results for Dirichlet problems with degenerated p-Laplacian. Opuscula Mathematica, 33,(3), 439-453.

[5] Cavalheiro, A. C. (2018). Topics on Degenerate Elliptic Equations, Lambert Academic Publishing, Germany.

[6] Drábek, P., Kufner, A., \& Nicolosi, F. (1997). Quasilinear Elliptic Equations with Degenerations and Singularities, Walter de Gruyter, Berlin.

[7] Bresch, D., Lemoine, J., \& Guíllen-Gonzalez, F. (2004). A note on a degenerate elliptic equation with applications for lakes and seas. Electronic Journal of Differential Equations (EJDE)[electronic only], 2004, 1-13.

[8] Colombo, M. (2017). Flows of Non-Smooth Vector Fields and Degenerate Elliptic Equations: With Applications to the Vlasov-Poisson and Semigeostrophic Systems. Publications on the Scuola Normale Superiore Pisa, 22, Pisa.

[9] Muckenhoupt, B. (1972). Weighted norm inequalities for the Hardy maximal function. Transactions of the American Mathematical Society, 165, 207-226.

[10] Torchinsky, A. (1986). Real-Variable Methods in Harmonic Analysis. Academic Press, San Diego.

[11] A. Kufner, A. (1985). Weighted Sobolev Spaces. John Wiley \& Sons.

[12] Heinonen, J., Kilpeläinen, T., \& Martio, O. (1993). Nonlinear Potential Theory of Degenerate Elliptic Equations, Oxford Math. Monographs, Clarendon Press.

[13] Gilbarg, D., \& Trudinger, M. S. (1983). Elliptic Partial Equations of Second Order, 2nd Ed., Springer, New York (1983).

[14] Chipot, M. (2009). Elliptic Equations: An introductory Course. Springer Science \& Business Media.

[15] Talbi, M., \& Tsouli, N. (2007). On the spectrum of the weighted p-Biharmonic operator with weight. Mediterranean Journal of Mathematics, 4(1), 73-86.

[16] Cuerva, J. G., \& De Francia, J. R. (1985). Weighted norm inequalities and related topics. North-Holland Mathematics Studies, 116.

[17] Turesson, B. O. (2000). Nonlinear Potential Theory and Weighted Sobolev Spaces. Lecture Notes in Math., Vol. 1736, Springer-Verlag.

[18] Kufner, A., \& Opic, B. (1984). How to define reasonably weighted Sobolev spaces. Commentationes Mathematicae Universitatis Carolinae, 25(3), 537-554.

[19] Fučik, S., John, O., \& Kufner, A. (1977). Function Spaces. Noordhoff International Publ., Leyden.

[20] Fabes, E. B., Kenig, C. E., \& Serapioni, R. P. (1982). The local regularity of solutions of degenerate elliptic equations. Communications in Partial Differential Equations, 7(1), 77-116.

[21] Zeidler, E. (1990). Nonlinear Functional Analysis and its Applications, Vol. II/B. Springer-Verlag.

[22] Zeidler, E. (1990). Nonlinear Functional Analysis and its Applications, Vol. I. Springer-Verlag.

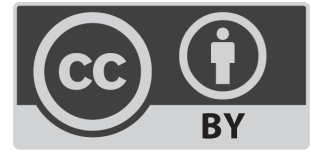

(C) 2020 by the authors; licensee PSRP, Lahore, Pakistan. This article is an open access article distributed under the terms and conditions of the Creative Commons Attribution (CC-BY) license (http://creativecommons.org/licenses/by/4.0/). 